\title{
Skilled Care Requirements for Elderly Patients After Coronary Artery Bypass Grafting
}

\author{
Brabmajee K. Nallamothu, MD, MPH, ${ }^{*}+\|$ Mary A. M. Rogers, PhD, MS, ${ }^{\dagger}$ Sanjay Saint, MD, MPH, ${ }^{* \dagger}$ \\ Laurence J. McMahon, Jr., MD, MPH, ${ }^{\dagger}$ Brant E. Fries, PhD, ${ }^{\ddagger \mathcal{S}}{ }^{\#}$ Samuel R. Kaufman, MSc, ${ }^{\dagger / l}$ and \\ Kenneth M. Langa, MD, PhD ${ }^{* \dagger \neq \| /}$
}

OBJECTIVES: To examine the extent to which elderly individuals use various skilled care facilities after coronary artery bypass grafting (CABG).

DESIGN: Retrospective cohort study.

SETTING: State of Michigan from 1997 to 1998.

PARTICIPANTS: Residents aged 65 and older enrolled in Medicare who underwent CABG.

MEASUREMENTS: Cumulative incidence of admission within 100 days of hospital discharge, relative risk (RR) of admission, readmission or extended stay at a skilled care facility, and length of stay in a skilled care facility.

RESULTS: Fifty percent of patients aged 80 and older used a skilled care facility after CABG, with most requiring admission to a skilled nursing facility (SNF) or readmission to an acute-care hospital within 100 days after discharge. $\mathrm{Pa}$ tients aged 80 and older had a significantly higher risk of admission to a SNF (adjusted RR $=3.3,95 \%$ confidence interval $(\mathrm{CI})=2.8-4.0)$ than did those aged 65 to 69 , as did patients aged 75 to 79 (adjusted $\mathrm{RR}=2.2,95 \% \mathrm{CI}=1.8$ 2.6) and those aged 70 to 74 (adjusted $R R=1.5,95 \%$ $\mathrm{CI}=1.3-1.8)$. The length of time spent in skilled care facilities significantly increased with age (mean days $=13.3$ for aged $65-69,16.9$ for $70-74,19.6$ for $75-79$, and 22.9 for 80 and older; $P<.001$ ).

CONCLUSION: Older patients are more likely to be admitted to a SNF, be readmitted to an acute-care hospital, and have longer institutional stays after CABG. When balancing the risks and benefits of CABG, physicians, patients,

From the *Health Services Research and Development Center of Excellence, Ann Arbor Veterans Affairs Medical Center, Ann Arbor, Michigan; ${ }^{\dagger}$ Department of Internal Medicine, Medical School, ${ }^{\ddagger}$ Institute for Social Research, ${ }^{\S}$ Department of Health Management and Policy, School of Public Health, University of Michigan, Ann Arbor, Michigan; "Patient Safety Enhancement Program, University of Michigan Health System, "SGIMHartford Collaborative Center for Research and Education in the Care of Older Adults, University of Michigan Health System, Ann Arbor, Michigan; and ${ }^{\#}$ Geriatric Research Education, and Clinical Center, Ann Arbor Veterans Affairs Medical Center, Ann Arbor, Michigan.

Address correspondence to Brahmajee K. Nallamothu, MD, MPH, Department of Internal Medicine-Cardiology, B1226 Taubman Center, Ann Arbor, MI 48109. E-mail: bnallamo@umich.edu

DOI: $10.1111 / \mathrm{j} .1532-5415.2005 .53356 . \mathrm{x}$ families, and policy-makers need to carefully consider the likelihood of follow-up institutional care in elderly patients. J Am Geriatr Soc 53:1133-1137, 2005.

Key words: aged; long-term care; skilled nursing facilities; health resources; Medicare; aftercare

$\mathrm{C}$ oronary artery bypass grafting (CABG) is effective at relieving symptoms and improving functional status in patients aged 80 and older. ${ }^{1-3}$ Although initial data suggested that CABG in those aged 80 and older was associated with exceptionally high in-hospital mortality rates and complications, more-recent evidence indicates that it is becoming increasingly safer to perform CABG in the very elderly. ${ }^{4,5}$ In-hospital and short-term mortality rates in those aged 80 and older now range from $5 \%$ to $14 \%$, and as a result, recent guidelines recommend that age alone should not be a contraindication to CABG. ${ }^{6}$

Despite these improvements in in-hospital outcomes after CABG, less is known about skilled care requirements in elderly patients after hospital discharge. This is particularly germane to professionals who counsel patients regarding postsurgical care and is useful in anticipating the healthcare services needed within the months after discharge from the hospital. Two previous studies demonstrated that patients aged 80 and older were at higher risk for discharge to skilled nursing facilities (SNFs) after CABG, but these investigations involved a small number of patients and did not differentiate between those requiring short-term rehabilitation and those requiring extended stays. ${ }^{7,8}$ Additional studies have shown that older patients are at higher risk for readmission to an acute-care hospital after CABG but did not report the extent of cross-facility use. ${ }^{9,10}$

To better understand the skilled care requirements of older patients after CABG, outcomes were evaluated in the Medicare population of Michigan. Answers to the following questions were of particular interest. What is the extent of post-CABG use of skilled care facilities with regard to type of facility and number of admissions in the elderly? 
Does use of these facilities vary with age after adjusting for demographics and comorbidities? What is the overall duration of stay in these facilities, including the likelihood of an extended stay, particularly in patients aged 80 and older?

\section{METHODS}

\section{Data Sources}

The following 1997/1998 Medicare Standard Analytic Files (SAFs) were obtained from the Centers for Medicare and Medicaid Services for the state of Michigan: inpatient, SNF, and hospice. These files contained demographic, diagnostic, and utilization data on hospitalizations, SNF stays, and hospice services. Medicare Part A completely covered all services provided during acute inpatient hospitalizations, whereas SNF care was covered for a period of up to 100 days after hospital discharge if the length of the hospital stay was at least 3 days. Direct transfers were covered as well as SNF admissions within 30 days of hospital discharge. Data were also obtained from the Medicare denominator and cross-reference files, which included information on vital status and changes in identification numbers during the study period.

\section{Study Population}

For these analyses, patients undergoing CABG between July 1, 1997, and September 22, 1998, were identified in the Medicare inpatient SAF using International Classification of Diseases, Ninth Revision, Clinical Modification (ICD-9CM) procedure codes 36.10 through $36.19 .^{11}$ These specific dates were selected to allow for a prehospital period of 6 months so as to identify comorbidities for case-mix adjustment and a follow-up period of 100 days. In cases in which patients underwent multiple CABG procedures during this time period, only the index surgery was used to determine immediate posthospitalization events. Only patients aged 65 and older at the time of CABG were included in these analyses. Patients residing at a SNF immediately before surgery were excluded $(n=7)$. Because the analysis focused on the use of skilled care after hospitalization, the analyses of postdischarge skilled care utilization were limited to those patients surviving to discharge.

Although the data were restricted to Michigan residents, they included information on healthcare utilization regardless of location. Thus, a Michigan resident who underwent CABG in Florida but 2 weeks later was admitted to a SNF in Michigan would be captured in the study population.

\section{Study Outcomes}

The primary outcomes of interest were any use of a SNF and total length of stay at a SNF within a 100-day follow-up period after hospital discharge. Extended SNF stays (i.e., longer than 30 days) and readmission to hospitals (acutecare, long-term care, rehabilitation, and psychiatric facilities) were also evaluated. "Complicated discharges" were defined as outcomes that included death, admission to a hospice program, an acute-care hospital readmission of any length, or a SNF stay of greater than 30 days within the 100day period after discharge from the CABG hospital stay.
The number of readmissions to various facilities and crossfacility usage within the 100-day period was recorded.

\section{Independent Variables}

Of primary interest was the association between a patient's age and the above outcomes. Patients were divided into four age categories for the analyses: 65 to 69,70 to 74,75 to 79 , and 80 and older. Covariates used in the analyses were sex, race (black vs nonblack), surgical priority (elective, urgent, or emergent), comorbid conditions, socioeconomic status, and hospital length of stay. Socioeconomic status was measured using the mean social security income of households in the patient's residential ZIP code area.

Comorbid conditions were identified using the Elixhauser method, which has been shown to be preferable to previously described risk-adjustment methods such as the Charlson-Deyo comorbidity score. ${ }^{12,13}$ The Elixhauser method was devised using administrative data to identify preexisting conditions using ICD-9-CM codes for secondary diagnoses. Data from the index hospital admission and all hospital admissions within a 6-month period before the CABG were used. Of the 30 Elixhauser comorbidities, 22 were present in more than $1 \%$ of the study subjects and were included in the adjusted models. These included congestive heart failure, cardiac arrhythmia, valvular heart disease, peripheral vascular disorders, hypertension, neurological disorders, chronic pulmonary disease, diabetes mellitus, renal failure, anemia, and depression.

\section{Statistical Analysis}

Chi-square tests for trend (Mantel extension test) were used to evaluate the relationship between age and patient characteristics (demographic, clinical, and healthcare utilization variables). One-way analysis of variance was used to evaluate differences in means across age categories. Cumulative incidence of outcomes (absolute risk) within the 100-day period after CABG was determined for all age categories. Population-averaged generalized estimating equations were used with the Poisson distribution and exchangeable correlation structure to account for clustering of data by hospital. ${ }^{14,15}$ Models were generated to assess the independent relationship between age and outcomes after adjustment for covariates using Huber/White/sandwich estimators of variance. ${ }^{16}$ Exponentiated coefficients were used to calculate relative risk (RR) ratios and their $95 \%$ confidence intervals (CIs). For all analyses, alpha was set at 0.05 and two-tailed tests were used.

\section{Human Subjects Protection}

Written permission from the Centers for Medicare and Medicaid Services was obtained for use of Medicare data files. The institutional review board of the University of Michigan Medical School also approved this study.

\section{RESULTS}

\section{Baseline Characteristics}

Nine thousand two hundred eleven Michigan residents who underwent isolated CABG during the study period were identified. Of these, 454 (4.9\%) died during hospitalization 
Table 1. Characteristics of Michigan Medicare Patients Who Underwent Coronary Artery Bypass Grafting, 1997-1998

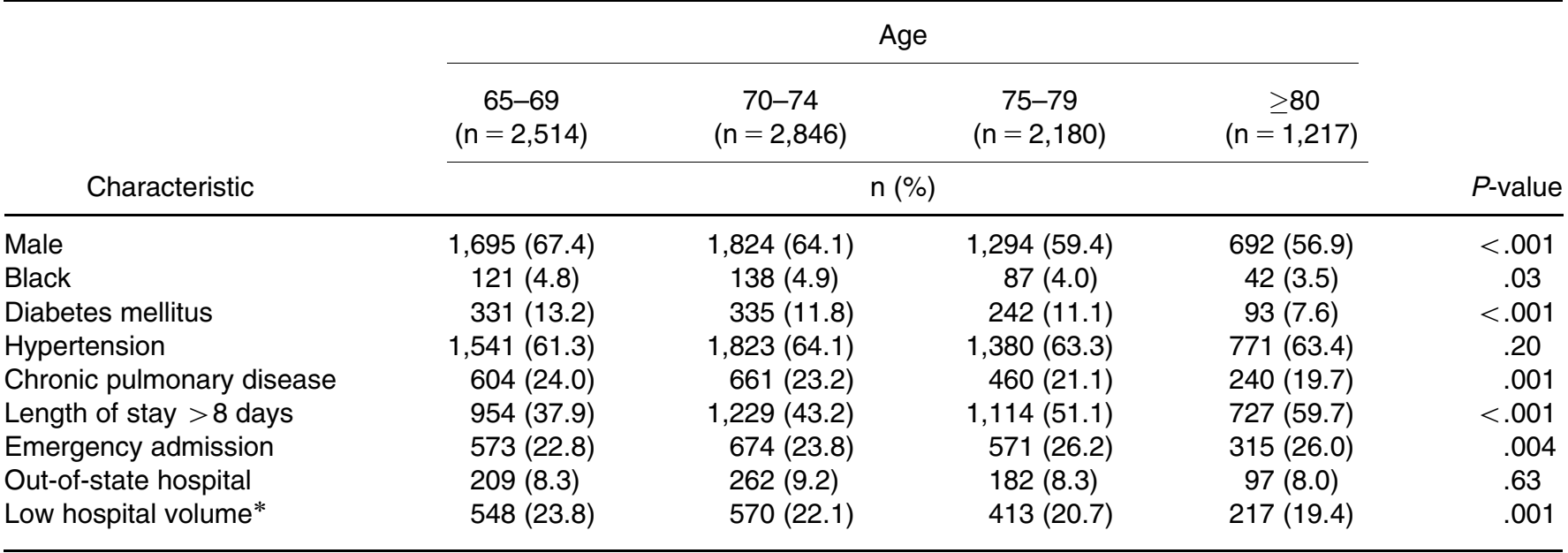

* Fewer than 400 coronary artery bypass grafting procedures performed at hospital annually.

after their CABG, leaving 8,757 patients for further analyses. Table 1 shows the baseline characteristics of this study population. Mean age \pm standard deviation was $73.7 \pm$ 5.4 , and $1,217(13.9 \%)$ patients were aged 80 and older. Most patients were men $(\mathrm{n}=5,505,62.9 \%)$, nonblack $(\mathrm{n}=8,369,95.6 \%)$, and received their CABG in Michigan $(\mathrm{n}=8,007,91.4 \%)$. In general, younger patients were more often male and black and had a higher prevalence of diabetes mellitus and chronic pulmonary diseases than older patients. Older patients had greater incidence of prolonged stays during their hospitalization for CABG (i.e., $>8$ days) and more emergent admissions. Finally, a greater proportion of younger patients underwent CABG at low-volume hospitals.

\section{Skilled Care Requirements After Hospital Discharge}

More than one-third of patients $(36.5 \%)$ used a SNF within 100 days of hospital discharge, and this need for additional care increased significantly with advancing age $(P<.001)$. Table 2 lists the distribution of skilled care requirements across facility types by age group. Half $(50.3 \%)$ of patients aged 80 and older required skilled care, with most requiring admission to a SNF $(23.0 \%)$ or readmission to an acutecare hospital $(38.9 \%)$ within 100 days after discharge. There was considerable cross-facility use, which is reflected in the "multiple facilities" category in Table 2.

Immediate discharge locations for CABG patients by age are given in Figure 1. Most patients were discharged home, but many of these patients were later readmitted to an acute-care hospital or a SNF. Of patients aged 80 and older, $71.9 \%$ were discharged home after cardiac surgery. Of these, $30.6 \%$ were later readmitted to an acute-care hospital, and $3.3 \%$ were admitted to a SNF. Readmission to an acute-care facility was also common $(41.0 \%)$ in patients aged 80 and older who were initially discharged to a SNF. Overall, 912 of the 8,757 patients $(10.4 \%)$ were readmitted to a hospital two or more times within the 100-day period, and $205(2.3 \%)$ were admitted to a SNF two or more times within the 100-day period.

During the 100 -day period after discharge, $23.0 \%$ of patients who were aged 80 and older, $12.9 \%$ of those aged

Table 2. Skilled Care of Michigan Medicare Patients Within 100 Days After Discharge from Hospitalization for Coronary Artery Bypass Grafting, 1997-1998

\begin{tabular}{|c|c|c|c|c|c|c|}
\hline \multirow[b]{3}{*}{ Facility Type } & \multicolumn{5}{|c|}{ Age } & \multirow[b]{3}{*}{$P$-value } \\
\hline & $\begin{array}{c}65-69 \\
(n=2,514)\end{array}$ & $\begin{array}{c}70-74 \\
(n=2,846)\end{array}$ & $\begin{array}{c}75-79 \\
(n=2,180)\end{array}$ & $\begin{array}{c}\geq 80 \\
(\mathrm{n}=1,217)\end{array}$ & Total & \\
\hline & \multicolumn{5}{|c|}{$n(\%)$} & \\
\hline Institutionalized skilled care & $716(28.5)$ & 986 (34.6) & $880(40.4)$ & $612(50.3)$ & $3,194(36.5)$ & $<.001$ \\
\hline Skilled nursing facility only & $40(1.6)$ & $102(3.6)$ & $125(5.7)$ & $140(11.5)$ & $407(4.6)$ & $<.001$ \\
\hline Acute-care hospital only & $534(21.2)$ & $613(21.5)$ & $454(20.8)$ & $227(18.7)$ & $1,828(20.9)$ & .10 \\
\hline Other hospitals only* & $42(1.7)$ & $62(2.2)$ & $76(3.5)$ & $55(4.5)$ & $235(2.7)$ & $<.001$ \\
\hline Multiple facilities ${ }^{\dagger}$ & $100(4.0)$ & $209(7.3)$ & 225 (10.3) & $190(15.6)$ & $724(8.3)$ & $<.001$ \\
\hline No institutionalized skilled care & $1,798(71.5)$ & $1,860(65.4)$ & $1,300(59.6)$ & $605(49.7)$ & $5,563(63.5)$ & $<.001$ \\
\hline
\end{tabular}

\footnotetext{
* Long-term care hospitals, rehabilitation hospitals or units, and psychiatric hospitals or units.
}

${ }^{\dagger}$ Skilled nursing facilities, acute-care hospitals, and other hospitals. 

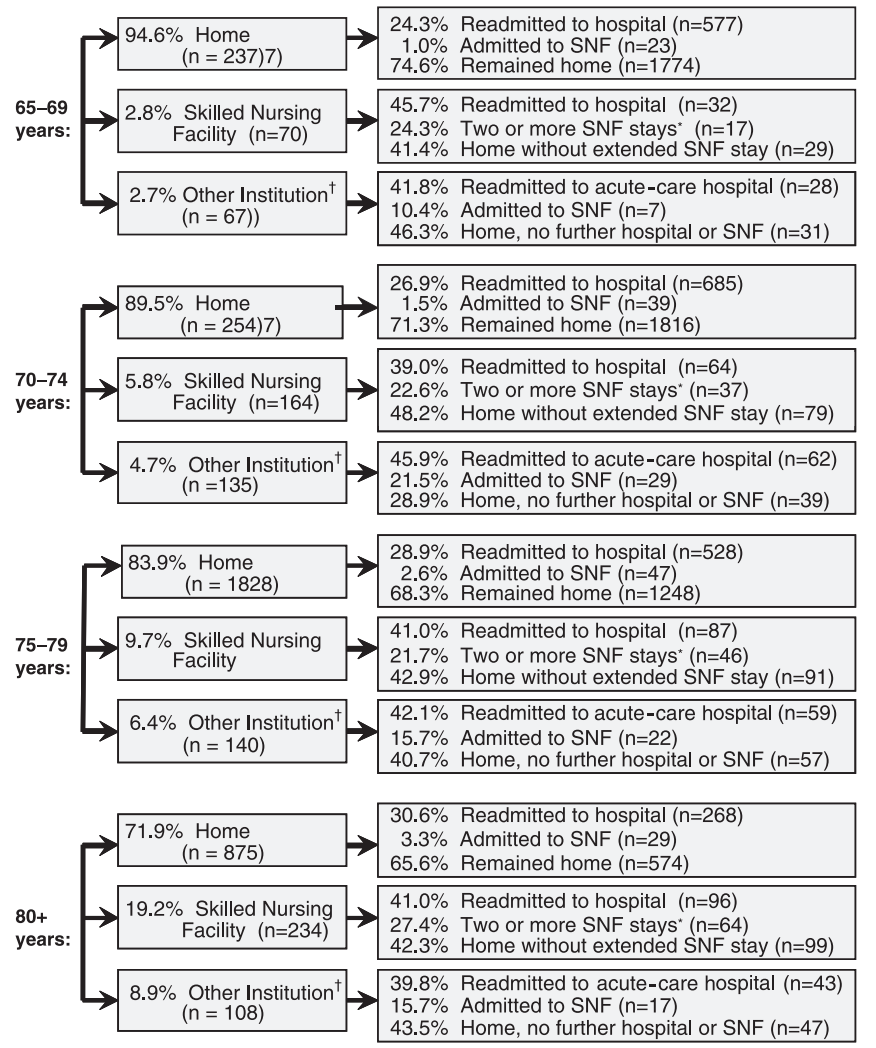

Figure 1. Skilled care utilization in Michigan Medicare patients within 100 days of hospital discharge from hospitalization for coronary artery bypass grafting, 1997/1998. Note: Percentages on the right do not always total $100 \%$ because the categories were not discrete. Some patients experienced more than two events listed. Some patients remained in the same institution throughout the entire 100-day period. Patients who received hospice services $(n=31)$ were excluded.

*Includes patients who were readmitted to an acute care hospital within the 100-day period.

$\dagger$ Other institutions include intermediate care facilities, acute care hospitals, and other types of institutions.

$\mathrm{SNF}=$ skilled nursing facility.

75 to $79,8.1 \%$ of those aged 70 to 74 , and $4.0 \%$ of those aged 65 to 69 were admitted to a SNF. Compared with that of patients aged 65 to 69 , the RR of admission was 3.3 $(95 \% \mathrm{CI}=2.8-4.0)$ for those aged 80 and older, 2.2 for those aged 75 to $79(95 \% \mathrm{CI}=1.8-2.6)$, and 1.5 for those aged 70 to $74(95 \% \mathrm{CI}=1.3-1.8)$, after adjustment for sex, race, surgical priority, Elixhauser comorbidities, social security income, and hospital length of stay. Readmission to an acute-care hospital during the 100-day period occurred in $26.9 \%$ of patients aged 65 to $69,31.1 \%$ of those aged 70 to $74,34.6 \%$ of those aged 75 to 79 , and $38.9 \%$ of those aged 80 and older. Similarly, complicated discharge occurred in $27.5 \%, 32.7 \%, 36.9 \%$, and $42.6 \%$ of the respective age groups. The risk of a complicated discharge was $40 \%$ greater in patients aged 80 and older than in those aged 65 to 69 (adjusted $\mathrm{RR}=1.4,95 \% \mathrm{CI}=1.3-1.5)$. The risk of death in the 100-day postdischarge period was $1.6 \%$ for those aged 65 to $69,2.5 \%$ for those aged 70 to $74,3.4 \%$ for those aged 75 to 79 , and $5.4 \%$ for those aged 80 and older $(P<.001)$.
Length of Stay at Skilled Care Facilities After Discharge

The mean length of stay in skilled care facilities (total time spent in various institutions) during the 100 -day period after hospital discharge increased with advancing age. For patients aged 80 and older, the mean length of stay \pm standard deviation was $22.9 \pm 23.1$ days, compared with 19.6 \pm 23.4 for those aged 75 to $79,16.9 \pm 21.7$ for those aged 70 to 74 , and $13.3 \pm 19.0$ for those aged 65 to 69 $(P<.001)$. Length of stay during the hospitalization for CABG was the single strongest predictor of an extended stay (>30 days) at a SNF. Those who remained in the hospital for more than 12 days had an approximately 11 times greater probability of an extended stay than those with hospital stays less than 8 days. Age also increased the risk of an extended stay; patients aged 80 and older were five times as likely to have an extended stay as those aged 65 to 69 (adjusted $\mathrm{RR}=5.1,95 \% \mathrm{CI}=3.4-7.8$ ).

\section{DISCUSSION}

Older age has a considerable effect on the likelihood of admission to a SNF and the use of other skilled care services after CABG, with half of all patients aged 80 and older requiring some skilled care after hospital discharge. Of patients transferred directly to SNFs, $27 \%$ had extended stays of more than 30 days. Approximately one-third of those aged 80 and older had extended stays. Other adverse outcomes, including death and hospital readmission, were also significantly more likely these older patients. All of these relationships remained significant after adjustment for multiple confounders, including sex, race, surgical priority, and comorbid conditions. These findings suggest that the likelihood for postdischarge skilled care requirements for the very elderly is substantial after CABG. The surgeon and patient must carefully consider this when assessing operative risk.

Substantial use of SNFs after CABG affects the decision to operate in two important ways. First, at the individual level, the need for a stay at a SNF is an important issue for elderly patients and their families. There remains a great fear among elderly patients and their families concerning the use of skilled nursing services, given their potential association with a loss of independence, prolonged stays, and decreased quality of life. ${ }^{17,18}$ Second, from a populationbased perspective, the use of these services after discharge dramatically increases resource utilization. It is important to assess the overall effects - not just in-hospital costsassociated with surgical revascularization in this age group, especially when making comparisons to less-invasive options such as percutaneous coronary intervention and medical therapy. These options may be limited in some patients who need to undergo CABG under emergent conditions and in whom alternatives are not possible.

There are likely several reasons for these findings. These include a higher risk of postoperative complications such as cerebrovascular accidents, renal insufficiency, and wound infections associated with sternotomy or saphenous vein graft harvesting in the very elderly. ${ }^{5}$ Even without overt cerebrovascular accidents, cognitive dysfunction after CABG is an increasingly recognized issue in very elderly patients and may also contribute to longer recovery times and additional nursing requirements. ${ }^{19}$ Another explanation for higher rates 
of discharge to SNFs in very elderly patients may be poorer social support systems, such as the absence of a living, independent spouse, than in younger patients. ${ }^{20}$

This study should be interpreted in the context of the following limitations. First, it evaluated only residents of Michigan. Although Michigan is diverse in terms of ethnicity and socioeconomic status, there is evidence that the use of skilled care services after acute-care hospitalizations varies substantially based on geographic region. ${ }^{21}$ However, the readmission rate to acute-care facilities in those aged 80 and older (38.9\% in 100 days) in this study was similar to previously reported rates. ${ }^{10}$ Second, it was not possible to capture all relevant factors associated with need for additional skilled care-such as functional status, cognitive status, and social support - in the multivariable models because of limitations of Medicare data files. These factors often are lacking in administrative data but have been shown to have a substantial influence on skilled care requirements in older people. ${ }^{20,22}$ Future research should focus on this area for early identification of those who are at the highest risk of needing additional skilled care. Similarly, it was not possible to account for some clinical covariates such as severity of coronary disease or left ventricular systolic function. Third, outcomes were evaluated during the 100-day period after hospital discharge. Examining the influence of age on longer-term outcomes, including permanent SNF requirements, is needed.

In conclusion, use of SNFs and acute-care hospitals within the 100-day period after hospital discharge after CABG is common in elderly patients. Half of patients aged 80 and older used a skilled care facility, with most requiring healthcare services at SNFs or acute-care hospitals. Physicians, patients, families, and policy-makers need to consider these findings when balancing the risks and benefits of CABG in individual patients. In addition, systems of care should be established with the expectation that a substantial number of elderly patients will require close follow-up after CABG, including the use of SNF care.

\section{ACKNOWLEDGMENTS}

Financial Disclosure(s): None

Author Contributions: Nallamothu (study concept and design, acquisition of data, analysis and interpretation of data, preparation of manuscript), Rogers (acquisition of data, analysis and interpretation of data, preparation of manuscript), Saint (study concept and design, acquisition of data, interpretation of data, preparation of manuscript), McMahon (study concept and design, acquisition of data, interpretation of data, preparation of manuscript), Fries (study concept and design, acquisition of data, interpretation of data, preparation of manuscript), Kaufman (acquisition of data, analysis and interpretation of data, preparation of manuscript), Langa (study concept and design, acquisition of data, interpretation of data, preparation of manuscript).

Sponsor's Role: Funding was provided by the Blue Cross and Blue Shield of Michigan Foundation and the University of Michigan Claude Pepper Older Americans Independence Center. Dr. Nallamothu is supported as a clinical scholar under a K12 grant from the National Institutes of Health (RR017607-01) and by the Mardigian
Foundation. Dr. Saint is supported by a Career Development Award from the Health Services Research and Development Program of the Department of Veterans Affairs and a Patient Safety Developmental Center Grant from the Agency for Healthcare Research and Quality (P20HS11540). Dr. Langa is supported by a Career Development Award from the National Institute on Aging (K08 AG19180), a New Investigator Research Grant from the Alzheimer's Association, and a Paul Beeson Physician Faculty Scholars in Aging Research award. Sponsors provided funding only; they did not participate in the design, methods, data collection, analyses, or preparation of the paper.

\section{REFERENCES}

1. Glower DD, Christopher TD, Milano CA et al. Performance status and outcome after coronary artery bypass grafting in persons aged 80 to 93 years. Am J Cardiol 1992;70:567-571.

2. Kaul TK, Fields BL, Wyatt DA et al. Angioplasty versus coronary artery bypass in octogenarians. Ann Thorac Surg 1994;58:1419-1426.

3. Alexander KP, Peterson ED. Coronary artery bypass grafting in the elderly. Am Heart J 1997;134:856-864.

4. Peterson ED, Cowper PA, Jollis JG et al. Outcomes of coronary artery bypass graft surgery in 24,461 patients aged 80 years or older. Circulation 1995; 9(Suppl 9):II85-II91.

5. Alexander KP, Anstrom KJ, Muhlbaier LH et al. Outcomes of cardiac surgery in patients $>$ or $=80$ years: Results from the National Cardiovascular Network. J Am Coll Cardiol 2000;35:731-738.

6. Eagle K, Guyton R, Davidoff R et al. Guidelines for coronary artery bypass graft surgery. A report of the American College of Cardiology/American Heart Association Task Force on Practice Guidelines. J Am Coll Cardiol 1999; 34:1262-1347.

7. Nallamothu BK, Saint S, Eagle KA et al. Coronary artery bypass grafting in octogenarians. Clinical and economic outcomes at community-based healthcare facilities. Am J Manag Care 2002;8:749-755.

8. Garza JJ, Gantt DS, Van Cleave $\mathrm{H}$ et al. Hospital disposition and long-term follow-up of patients aged $>I=80$ years undergoing coronary artery revascularization. Am J Cardiol 2003;92:590-592.

9. Hannan EL, Racz MJ, Walford G et al. Predictors of readmission for complications of coronary artery bypass graft surgery. JAMA 2003;290:773-780.

10. Jarvinen $\mathrm{O}$, Huhtala $\mathrm{H}$, Laurikka J et al. Higher age predicts adverse outcome and readmission after coronary artery bypass grafting. World J Surg 2003; 27:1317-1322.

11. International Classification of Diseases, 9th Revision, Clinical Modification, 5th Ed, Color Coded. Los Angeles: Practice Management Information Corp., 1997.

12. Elixhauser A, Steiner C, Harris DR et al. Comorbidity measures for use with administrative data. Med Care 1998;36:8-27.

13. Southern DA, Quan H, Ghali WA. Comparison of the Elixhauser and Charlson/Deyo methods of comorbidity measurement in administrative data. Med Care 2004;42:355-360.

14. Liang KY, Zeger SL. Longitudinal data analysis using generalized linear models. Biometrika 1986;73:13-22.

15. Zou G. A modified Poisson regression approach to prospective studies with binary data. Am J Epidemiol 2004;159:702-706.

16. Rogers WH. Regression standard errors in clustered samples. Stata Techn Bull 1993;13:19-23.

17. American Association of Retired Persons. These Four Walls... Americans 45+Talk About Home and Community. Available at: http://research.aarp.org/ il/four_walls.html Accessed July 9, 2004

18. Coles R. Old and on Their Own. New York: W.W. Norton, 1997.

19. Scarborough JE, White W, Derilus FE et al. Neurologic outcomes after coronary artery bypass grafting with and without cardiopulmonary bypass. Semin Thorac Cardiovasc Surg 2003;15:52-62.

20. Banaszak-Holl J, Fendrick AM, Foster N et al. Predicting nursing home admission. Estimates from a seven-year follow-up of a nationally representative sample of older Americans. Alzheimer Dis Assoc Disord 2004;18:83-89.

21. Cohen MA, Tumlinson A. Understanding the state variation in Medicare home health care. The impact of Medicaid program characteristics, state policy, and provider attributes. Med Care 1997;35:618-633.

22. Langa KM, Chernew M, Kabeto $M$ et al. National estimates of the quantity and cost of informal caregiving for the elderly with dementia. J Gen Intern Med 2001;16:770-778. 